\title{
P01-55
}

\section{THE EFFECT OF TOPIRAMATE IN THE TREATMENT OF OPIOID (HEROIN) WITHDRAWAL}

N. Mokhber, A. Soltanifar, M. Talebi

Mashad University of Medical Sciences, Mashad, Iran

Objective: This study was carried out on 149 of Heroin dependent patient that admission for detoxification.

Design: This study is a 10 days double blind placebo-controlled trial .

Place \& duration of study: This study is a 10 days double blind placebo-controlled trial which was carried out on 149 of Heroin dependent patient that admission for detoxification in Mashhad Ebne-sina hospital in 1385-1386 Subjects were randomly divided into topiramate and placebo detoxification groups.

Subjects \& methods: Topiramate group receive topiramate (max $200 \mathrm{mg}$ ) for 10 days . The placebo group received usual regimen and placebo. Withdrawal symptoms were assessed by Subjective and Objective Opiate Withdrawal Scale in days 1, 3, 5, 7, 10.information $s$ analyzed by chi-square test for qualities variables and T-test \& Mann-Whitney for quantities variables in SPSS software.

Results: 22 subjects were excluded from the study. Both groups had no significant differences in age, sex, education, number of previous withdrawal and past history of other drug use. Result indicates decrease in objective withdrawal symptom significant in $3(p=0.000), 5(p=0.004)$ and $7(p=0.026)$ day and subjective withdrawal symptom significant in $3(p=0.000)$ day in topiramate groups.

Conclusion: Topiramate can use for control of withdrawal symptom in patient with Heroin dependency.

Key words: withdrawal, detoxification, topiramate, Opium, Heroin. 